Check for updates

Cite this: RSC Adv., 2019, 9, 14621

\title{
Effects of membrane thickness on the performance of ionic polymer-metal composite actuators $\uparrow$
}

Received 7th March 2019 Accepted 29th April 2019

DOI: $10.1039 / c 9 r a 01751 h$

rsc.li/rsc-advances
Chungik Oh, ${ }^{a}$ Suran Kim, ${ }^{a}$ Hongjun Kim, ${ }^{a}$ Gun Park, ${ }^{a}$ Jaegyu Kim, ${ }^{a}$ Jeongjae Ryu, ${ }^{a}$
Panpan Li, ${ }^{a}$ Sunghwan Lee, (D) ${ }^{b}$ Kwangsoo No ${ }^{a}$ and Seungbum Hong (ID) *ac

In this study, we report the effects of Nafion thickness on the performance of ionic polymer-metal composite (IPMC) actuators. We analyzed the actuation properties of the IPMC actuators, such as displacement and tip force, under external voltage, as a function of their thickness. In order to understand the relationship between thickness and actuation properties, we developed a semiquantitative model of voltage induced ionic diffusion and its contribution to bending of the Nafion cantilever. Furthermore, we investigated the mechanical properties of the Nafion membranes at submicro scale as well as bulk scale, using atomic force microscopy (AFM) and tensile test. The results of the two methods indicated opposite trends of elastic modulus and crystallinity as a function of thickness. We hypothesized that the hot-pressed Nafion was composed of three layers with different crystallinity. Our results suggest that for a high performance IPMC actuator, we need better control of the annealing temperature gradient.

\section{Introduction}

Ionic polymer metal composite (IPMC) actuators which exhibit electromechanical transduction have attracted significant interest for soft actuator applications such as artificial muscles, biomimetic robots, and biomedicines. The general IPMC structure is electrode/membrane (ionic polymer)/electrode, and overall device performance is, in large part, dependent on the properties of the membrane. Nafion (perfluorosulfonate) ${ }^{1-5}$ Flemion (perfluorocarboxylate $)^{6-8}$ and other perfluorinated compounds such as aciplex (perfluorosulfonate) and hyflon (perfluoroalkoxy) are widely employed as a membrane for IPMC actuators, due to their low weight, flexibility and large displacement under low driving voltage $(<5 \mathrm{~V}) .^{5,9}$ Among these ionic polymers, Nafion has gained particular attention because of its excellent chemical resistance, a higher working temperature as compared to other polymers and high ion conductivity. ${ }^{3-5,7-11}$

Many studies have been carried out to improve the performance of IPMC actuators using Nafion composites or other polymers, including carbon materials such as graphene, ${ }^{\mathbf{1 2 , 1 3}}$ multi-walled carbon nanotubes (MWCNT), ${ }^{\mathbf{1 4 , 1 5}}$ and oxide particles such as $\mathrm{BaTiO}_{3}{ }^{16}$ The polymers investigated

${ }^{a}$ Department of Materials Science and Engineering, KAIST, Daejeon 34141, Korea. E-mail: seungbum@kaist.ac.kr

${ }^{b}$ School of Engineering Technology, Purdue University, West Lafayette, IN 47907, USA ${ }^{c}$ KAIST Institute for the NanoCentury, Daejeon 34141, Korea

$\dagger$ Electronic supplementary information (ESI) available. See DOI: 10.1039/c9ra01751h to replace Nafion include kraton, ${ }^{17}$ poly-vinylidene fluoride (PVDF)/polyvinyl pyrrolidone (PVP)/polystyrene sulfuric acid (PSSA) ${ }^{18}$ and a series of sulfonated polysulfone (SPSU). ${ }^{19}$

Another method to improve the performance of IPMC actuators is to replace the electrode materials. Conventionally, IPMC actuators are fabricated by electroless plating platinum (Pt) electrodes onto a Nafion membrane. ${ }^{20}$ However, this method is time consuming and expensive. In addition, the metal electrode experiences cracking during operation, which causes performance degradation. Therefore, simpler electrode deposition methods such as dip coating, ${ }^{21}$ and replacing the metal materials with conducting material/Nafion composites in the electrodes have been studied. Carbon materials such as graphene ${ }^{\mathbf{1 3 2 1}}$ and MWCNT have been used as the conducting materials. Other metals, such as gold $(\mathrm{Au}),{ }^{22,23}$ palladium $(\mathrm{Pd}),{ }^{22-24}$ and conductive polymers such as poly(3,4-ethylenedioxythiophene) polystyrene sulfonate (PEDOT:PSS) ${ }^{25}$ have also been used as electrodes.

However, basic factors, such as the thickness dependence of performance, are not well understood at multiscale. The thickness of the Nafion membranes is closely related to the performance of the actuators. Therefore, we systematically analyzed actuation performance under an external voltage to find how Nafion membrane thickness affects the performance of IPMC actuators. In addition, to understand the properties of hot-pressed Nafion membrane, we investigated the crystallinity and mechanical properties of Nafion membrane using atomic force microscopy (AFM) and tensile tests. 


\section{Experimental}

To make Nafion membranes (NR212, N117, Dupont) with different thicknesses, the Nafion membranes were hot-pressed at $150{ }^{\circ} \mathrm{C}$ at a uniaxial pressure of $30 \mathrm{MPa}$ for $30 \mathrm{~min}$. The reason we chose $150{ }^{\circ} \mathrm{C}$ is that the membrane spreads out at temperatures over $160{ }^{\circ} \mathrm{C}$. This may be related to the two different glass transition temperatures, at $100-120{ }^{\circ} \mathrm{C}$ and $190-$ $240{ }^{\circ} \mathrm{C}^{.26-28}$ We set the pressing time to $30 \mathrm{~min}$ because there was no significant difference when we varied the time up to $5 \mathrm{~h}$.

The IPMC actuators (width/length $=1 \mathrm{~cm} / 3 \mathrm{~cm}$ ) were fabricated using a direct-assembly-process (DAP). In order to increase the interfacial area and enhance adhesion between the Nafion membrane and electrodes, the surface of the Nafion was roughened using sandpaper (\#1200). For the electrode material, multi-wall carbon nanotubes (MWCNTs, Applied Carbon Nano Technology Co.) were utilized. The solution of MWCNTs, Nafion dispersion and isopropyl alcohol was prepared by stirring the mixture for $1 \mathrm{~h}$, and then the solution was painted on both sides of the cast Nafion membranes, followed by drying at $120{ }^{\circ} \mathrm{C}$ for $20 \mathrm{~min}$. To further enhance adhesion between the electrodes and Nafion films, the samples were hot-pressed at $110{ }^{\circ} \mathrm{C}$ at a uniaxial pressure of $10 \mathrm{MPa}$ for $10 \mathrm{~min}$ as shown in ESI Fig. S1. $\dagger$

Atomic force microscopy (AFM) analysis was performed to evaluate the effect of the roughening treatment on the surface and ANOVA test was performed to verify the reproducibility and repeatability of the roughening process based on the results [see ESI Fig. S2 and Table S1†].

The performance of the IPMC actuators was systematically evaluated. The displacement of the devices was monitored at an ac voltage of $3 \mathrm{~V}, 0.5 \mathrm{~Hz}$ using a displacement measurement sensor (OD85-20T1, SICK), whose laser beam was illuminated perpendicular to the tip of the devices. The distance $\left(d_{0}\right)$ between the laser point and fixed end point of IPMC strip is about $86 \mathrm{~mm} \pm 19 \mathrm{~mm}$. The displacement $(d)$ was measured by subtracting the measured distance from the initial value of distance as shown in ESI Fig. S3(a) and (c). $\uparrow$ A load cell (GSO-30, Transducer Techniques) was used to measure the tip force of the actuators. The IPMC actuators were attached to one end of the pin of the load cell in an initial condition (zero displacement) as shown in ESI Fig. S3(b). $\dagger$

To measure the elastic modulus of the Nafion membrane in nano-micro scale, we used an atomic force microscopy (AFM) indentation method as shown in ESI Fig. S4. $\dagger$

\section{Results and discussion}

We surveyed the reported maximum tip forces that had been observed when a dc bias voltage was applied across the Nafion membrane as a function of its thickness as shown in Fig. 1. 1.29-31 Although variations were observed between different groups, the general trend shows that as the thickness of the Nafion membrane increases, the tip force tends to increase. Many groups attributed this trend to the increase in bending stiffness as a function of IPMC thickness. The bending stiffness (EI) is proportional to the cube of the

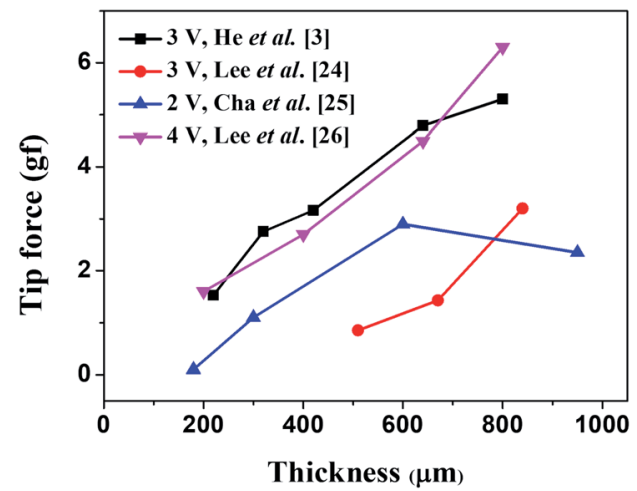

Fig. 1 The tip force versus thickness of Nafion membranes under dc voltage, as reported in the literature, are plotted following review. In general, the tip force tends to increase as the Nafion membrane becomes thicker.

thickness for rectangular cantilever shapes, which is the multiplication of the elastic modulus and moment of inertia as shown in eqn (1).

$$
E I=E \frac{w t^{3}}{12}
$$

where $E$ is the elastic modulus, $I$ is the moment of inertia, and $w$ and $t$ are the width and thickness of the IPMC actuators, respectively. ${ }^{3,30,31}$ However, it is not well understood why the tip force does not scale with the cube of the thickness and instead scales linearly with the thickness in most cases.

In order to quantitatively understand the thickness dependence of both maximum tip force and maximum displacement, we fabricated Nafion membrane-based cantilevers with different thickness from $52 \mu \mathrm{m}$ to $714 \mu \mathrm{m}$. Fig. 2 shows the maximum displacement (blue line) under an ac voltage of $3 \mathrm{~V}$, $0.5 \mathrm{~Hz}$ and the maximum tip force (red line) under a dc voltage of $3 \mathrm{~V}$, as a function of the Nafion membrane thickness. As the thickness of the Nafion membrane increased, the maximum displacement decreased, and the maximum tip force increased, in a trend similar to Fig. 1.

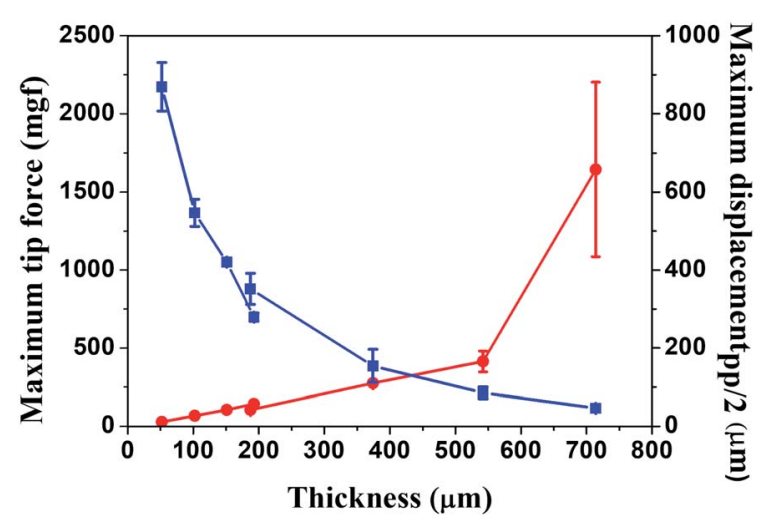

Fig. 2 The maximum displacement under $3 \mathrm{~V}, 0.5 \mathrm{~Hz}$ ac bias (blue line) and maximum tip force under $3 \mathrm{~V}$ dc bias (red line) of IPMC actuators plotted for varying Nafion membrane thicknesses. As thickness increases, displacement decreases and tip force increases. 
The reason for the smaller absolute values of maximum tip force for the same thickness may have to do with differences in the dimensions (width and length) of the IPMC actuators. He et $a l .{ }^{3}$ and Lee et al. ${ }^{29}$ operated theirs under a dc voltage of $3 \mathrm{~V}$, with dimensions of $5 \mathrm{~mm} \times 20 \mathrm{~mm}$ and $5 \mathrm{~mm} \times 30 \mathrm{~mm}$, respectively. Cha et $a l .{ }^{30}$ and Lee $e t ~ a l .{ }^{31}$ operated IPMC actuators under de voltages of $2 \mathrm{~V}$ and $4 \mathrm{~V}$, respectively, with dimensions (width and length) of $5 \mathrm{~mm} \times 30 \mathrm{~mm}$ and $5 \times 10$ $\mathrm{mm}$, respectively.

Another reason may be that the electrodes they used in their IPMC actuators were different. They deposited platinum (Pt) by electroless plating,,$^{3,29-31}$ but we used MWCNTs/Nafion composite as electrodes.

In order to explain the migration of ions and the volume change of the Nafion membrane with the external applied voltage, we provide a schematic diagram in Fig. 3(a). We assume that when we apply voltage there will be an electrical driving force in the form of electric field $E$ setting the ionic current $J$,

$$
J=\sigma E=-\sigma \frac{\partial V}{\partial z}
$$

where $\sigma$ is the electrical conductivity of Nafion. The ionic current $J$ should be counter-balanced by diffusion current $J_{\mu}$ driven by the electrochemical potential gradient in the form of ion concentration gradient $\frac{\partial C}{\partial z}$,

$$
J_{\mu}=-D \frac{\partial C}{\partial z}
$$

where $D$ is the diffusion coefficient of $\mathrm{H}^{+}$ions in Nafion. If we use the fact that $J+J_{\mu}=0$ for equilibrium,

$$
\sigma \frac{\partial V}{\partial z}=-D \frac{\partial C}{\partial z}
$$

Therefore,

$$
\sigma \Delta V=-D \Delta C+\text { consant }
$$

So for given $\Delta V, \sigma$, and $D, \Delta C$ should be constant regardless of the thickness. Since we assume that the $\mathrm{H}^{+}$concentration is

(a)

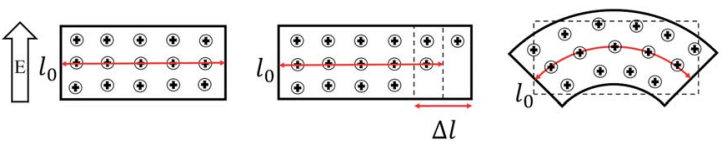

(b)

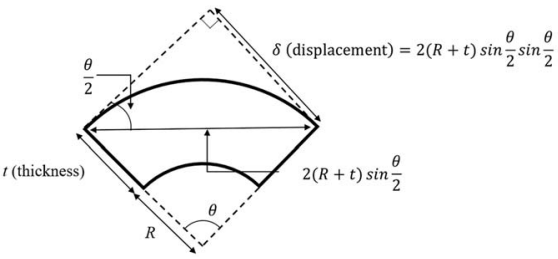

Fig. 3 (a) Schematic of the bending motion of IPMC actuators due to ions in the Nafion membrane. The ions are rearranged by an applied electric field $(E)$. (b) Schematic used to mathematically analyse the relationship between the Nafion membrane thickness $(t)$ and displacement $(\delta)$. uniform before the application of voltage, $\Delta C=0$ when $\Delta V=0$. Therefore, the integration constant will be zero in our case, and eqn (5) will change to eqn (6) as shown below.

$$
\sigma \Delta V=-D \Delta C
$$

The meaning of eqn (6) is that when we apply the same voltage $(\Delta V)$ across the Nafion membrane, the ionic concentration difference $(\Delta C)$ across the membrane will be the same regardless of the thickness as shown in Fig. 3(a).

Since the volume of the Nafion linearly scales with the ionic concentration according to Vegard's law, ${ }^{32}$ we assume that the length of the Nafion linearly scales with $\Delta C$. In our studies, since we applied the same voltage across the Nafion membrane, the difference $(\Delta l)$ between the expanded part due to the addition of ions and the contracted part due to the reduction of ions should be the same regardless of thickness, as shown in Fig. 3(a). Furthermore, because the membrane should keep its components consistent without an abrupt change in dimension, it should bend as shown in Fig. 3(a).

Fig. 3(b) shows the shape of the IPMC actuators bent by the movement of ions. The displacement $(\delta)$ induced by the difference in volume expansion and contraction is derived as follows.

$$
\begin{gathered}
\delta=2(R+t) \frac{\theta^{2}}{4}=\frac{1}{2}(R+t) \theta^{2}, \quad \text { if } \theta \text { is small } \sin \frac{\theta}{2} \approx \frac{\theta}{2} \\
(R+t) \theta-R \theta=t \theta=\Delta l, \theta=\frac{\Delta l}{t} \\
\delta=\frac{1}{2}(R+t) \frac{\Delta l^{2}}{t^{2}}
\end{gathered}
$$

As mentioned above, we assume that there is no change in the length of the middle side, i.e. $\left(R+\frac{t}{2}\right) \theta=l_{0}$.

So,

$$
\begin{gathered}
R=\frac{l_{0}}{\theta}-\frac{t}{2}=\frac{l_{0}}{\Delta l} t-\frac{t}{2} \\
\delta=\frac{1}{2}\left(\frac{l_{0}}{\Delta l} t-\frac{t}{2}+t\right) \frac{\Delta l^{2}}{t^{2}}=\frac{1}{2}\left(\frac{l_{0}}{\Delta l}+\frac{1}{2}\right) \frac{\Delta l^{2}}{t}=\frac{1}{2}\left(l_{0} \Delta l+\Delta l^{2}\right) \frac{1}{t}
\end{gathered}
$$

According to the aforementioned from eqn (2) to (6), we can verify that $\Delta l$ will be constant regardless of the thickness when the same voltage is applied. Based on these conclusions, we also calculated the displacement of the IPMC actuator as a function of thickness from eqn (7) to (11). The conclusions of the two derived equations eqn (6) and (11) show that the displacement $(\delta)$ is inversely proportional to the thickness of the Nafion membrane $(t)$ because $l_{0}$ and $\Delta l$ should be constant regardless of the thickness when the same voltage is applied. We fit the theoretical results based on our experimental data, as shown in Fig. 4.

In order to understand the trend observed in Fig. 2, we conducted an experiment to measure the elastic modulus both 


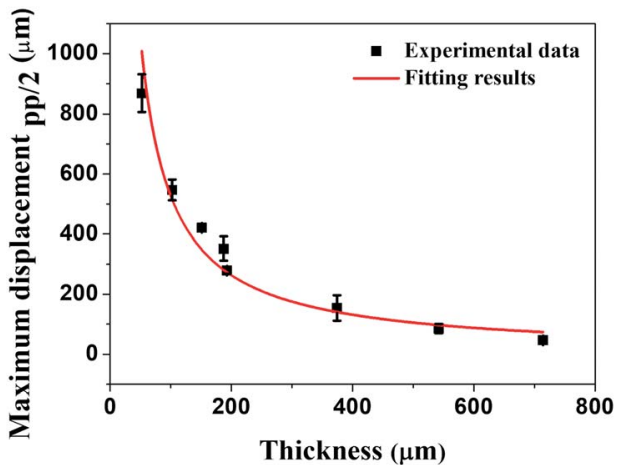

Fig. 4 The dotted data show that the maximum displacement of the IPMC actuators, and the solid red line is a fitting line using eqn (11).

at macroscale and micro/nanoscale. We measured the forcedistance curves using atomic force microscopy (AFM), collected the indentation curves over an $80 \mu \mathrm{m} \times 80 \mu \mathrm{m}$ area of Nafion membrane and mapped the elastic modulus as shown in ESI Fig. S5 $†$ by Hertzian model fitting of the force-indentation depth curve at each point. We indented with a loading force of about $8.11 \mu \mathrm{N}$ at all points and fitted the loading curve using eqn $(12)^{33}$

$$
F=\frac{2}{\pi} \tan \alpha E_{\mathrm{r}} \delta^{2}
$$

where $F$ is the force, $\delta$ is the indentation depth, $\alpha$ is the half angle of the AFM tip and $E_{\mathrm{r}}$ is the reduced elastic modulus, which takes into account both tip and sample deformation. The indentation depth $(\delta)$ was calculated by the difference between the $Z$ sensor value $(Z)$ and the cantilever deflection value $(x)$ measured by AFM, and the force $(F)$ was calculated by Hooke's law, which is a multiplication of the measured spring constant and deflection of the cantilever. From the measured $E_{\mathrm{r}}$, we can calculate the elastic modulus of the Nafion membrane using eqn (13)..$^{33-36}$

$$
\frac{1}{E_{\mathrm{r}}}=\frac{1-v_{\mathrm{s}}^{2}}{E_{\mathrm{s}}}+\frac{1-v_{\mathrm{t}}^{2}}{E_{\mathrm{t}}}
$$

where $E_{\mathrm{s}}$ and $v_{\mathrm{s}}$ are the sample elastic modulus and Poisson's ratio respectively, and $E_{\mathrm{t}}$ and $v_{\mathrm{t}}$ are the elastic modulus and Poisson's ratio, respectively, of the AFM tip. By comparing the elastic modulus map with the topography image, it is possible to identify the difference in elastic modulus between particles and particle boundaries. However, because the current spatial resolution is not sufficient to distinguish the difference between particles and particle boundaries, we leave this to future research.

In Fig. 5, we plotted the elastic modulus of Nafion membranes composed of both NR212 and N117 as measured by tensile test (red lines) and AFM indentation (blue lines). From the results of the tensile tests, the overall trend was that the elastic modulus decreased as the thickness increased except for the single sheet of NR212 Nafion membrane.

This trend is opposite to the one obtained using AFM indentation, whose results indicated that the elastic modulus

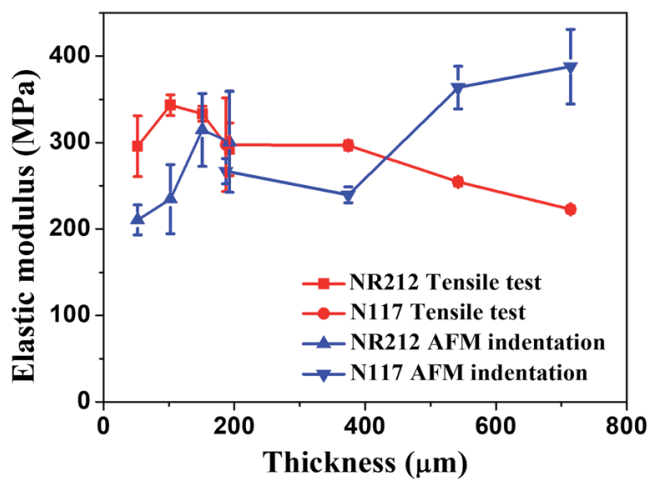

Fig. 5 The elastic modulus of Nafion membranes NR212 and N117, measured by tensile test (red lines) and AFM indentation (blue lines).

increased as the thickness increased. AFM indentation is surface sensitive, so the elastic modulus obtained by AFM reflects the characteristics of the thin surface layer. However, the tensile test measures the whole structure, so the larger volume inside the Nafion layer will contribute more to the elastic modulus. Given this, if the trends in the elastic modulus of the surface layer is different than the modulus of the inner part, we can expect to see an opposing trend in elastic modulus, as depicted in Fig. 5.

In order to see if this was really the case, we characterized the crystallinity of the Nafion layer using X-ray diffractometer. Fig. 6(a-c) shows the XRD patterns for each Nafion membrane.

(a)
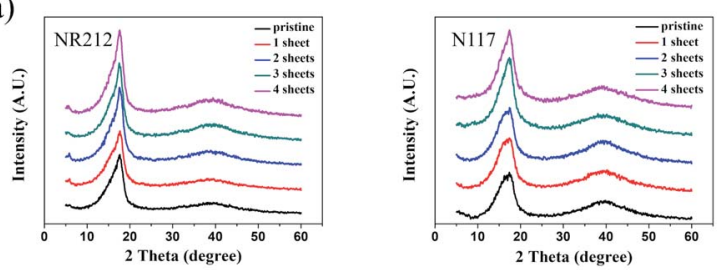

(b)
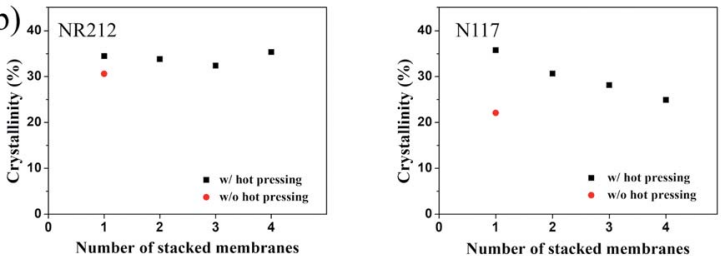

(c)

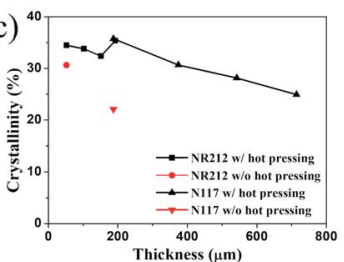

(d)

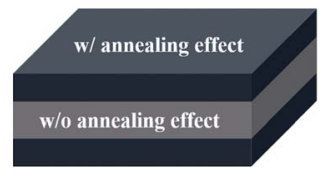

Fig. 6 (a) X-ray diffraction patterns and (b) crystallinity of Nafion membranes, prepared with 1 sheet to 4 sheets, without hot pressing (red dot) and with hot pressing (black dots) and (c) the crystallinity of the Nafion membranes as a function of thickness. (d) Schematic of the effect on the surface and inside the Nafion membrane when hotpressed. 
We calculated the crystallinity of each membrane by deconvoluting the amorphous and crystalline peaks. ${ }^{37,38}$ In this calculation, we assumed that the crystalline region of the Nafion, the carbon backbone (PTFE), was perfectly crystalline and that the sulfonic acid groups represented disordered regions.

The hot-pressed Nafion membranes (black dots) had increased crystallinity compared to the one prepared without hot pressing (red dot) for both NR212 and N117. Our data attests to the fact that hot pressing has a significant impact on the crystallization of the Nafion membrane we studied. In addition, the overall crystallinity decreased as the thickness of the Nafion membrane increased, which could potentially explain the decrease in elastic modulus measured by the tensile test. However, it is expected that the crystallinity of the surface layer would increase compared with the as-fabricated membrane, and that the thickness of the surface layer would also increase as the thickness of the Nafion membrane increased. This may explain the increasing trend in elastic modulus measured by the AFM indentation test.

It should be noted, though, that the hot-pressing conditions may have to be varied to yield the same results, depending on the membrane. Fig. 6(c) shows the crystallinity of the Nafion membrane after hot-pressing as a function of thickness. The overall trend is that the crystallinity decreases as the thickness increases. We developed a hypothesis to explain this trend, as illustrated in Fig. 6(d).

Fig. 6(d) shows the surface and internal states of the Nafion membrane after the hot-pressing. We hypothesize that during the hot-pressing, an annealing effect occurs from the surface to the inside of the Nafion membrane, leading to a sandwich structure. In order to produce a uniformly annealed layer, one has to increase the pressure and temperature of the hotpressing. This is difficult, considering the fact that the conditions of annealing time and temperature to achieve full annealing are different for each thickness. Therefore, we suggest that one should use an oven to apply uniform heat, which will result in a Nafion membrane with uniform crystallinity.

\section{Conclusions}

In summary, we fabricated Nafion membranes with different thickness using the hot pressing method, and studied the dependence of elastic modulus on thickness and its relationship with crystallinity. Under the same hot-pressing process conditions $\left(150{ }^{\circ} \mathrm{C}, 30 \mathrm{MPa}, 30 \mathrm{~min}\right)$, the crystallinity of the Nafion membrane increased compared to that of the Nafion membrane without hot-pressing treatment. However, the degree of crystallinity decreased as the thickness increased (as the number of stacked films increased) and approached the value of the Nafion membrane without hot-pressing treatment.

Furthermore, we found that the macroscopic elastic moduli of the Nafion membranes with different thickness, as well as the Nafion membranes without hot-pressing, were strongly coupled to their degrees of crystallinity. Finally, we hypothesized that the change in crystallinity of the Nafion membranes during hotpressing occurred from the surface inward, based on the elastic modulus mapping using atomic force microscopy (AFM). Our results can provide insight into the complex mechanical properties of Nafion membranes.

\section{Conflicts of interest}

There are no conflicts to declare.

\section{Acknowledgements}

This work was supported by the KUSTAR-KAIST Institute, KAIST, Korea, the Korea Research Fellowship Program funded by the National Research Foundation of Korea (No. 2017H1D3A1A01054478), the KAIST GCORE (Global Center for Open Research with Enterprise) grant funded by the Ministry of Science and ICT (Project \#N11190026), Basic Science Research Programs (No. 2018R1A2B6002194) through the NRF, Korea funded by the Ministry of Science and ICT, and Big Sciences Research Program (No. 2017M1A2A2044498) through the NRF, Korea funded by the Ministry of Science and ICT.

\section{Notes and references}

1 M. Shahinpoor and K. J. Kim, Smart Mater. Struct., 2001, 10, 819-833.

2 D. Pugal, K. Jung, A. Aabloo and K. J. Kim, Polym. Int., 2009, 59, 279-289.

3 Q. He, M. Yu, L. Song, H. Ding, X. Zhang and Z. Dai, J. Bionic Eng., 2011, 8, 77-85.

4 R. Tiwari and E. Garcia, Smart Mater. Struct., 2011, 20, 083001.

5 B. Bhandari, G.-Y. Lee and S.-H. Ahn, Int. J. Precis. Eng. Man., 2012, 13, 141-163.

6 J. Wang, C. Xu and M. Taya, J. Mater. Res., 2006, 21, 20182022.

7 J. Wang, H. Sato, C. Xu and M. Taya, J. Appl. Phys., 2009, 105, 083515.

8 J. Wang, C. Xu, M. Taya and Y. Kuga, Smart Mater. Struct., 2007, 16, S214-S219.

9 S. Mohsen and J. K. Kwang, Smart Mater. Struct., 2001, 10, 819.

10 D. Pugal, K. Jung, A. Aabloo and K. J. Kim, Polym. Int., 2010, 59, 279-289.

11 J. Wang, C. Xu, M. Taya and Y. Kuga, J. Mater. Res., 2006, 21, 2018-2022.

12 L. Naji, M. Safari and S. Moaven, Carbon, 2016, 100, 243-257. 13 L. Zu, Y. Li, H. Lian, Y. Hu, W. Chang, B. Liu, Y. Liu, X. Ao, Q. Li and X. Cui, Macromol. Mater. Eng., 2016, 301, 10761083.

14 J. Ru, Y. Wang, L. Chang, H. Chen and D. Li, Smart Mater. Struct., 2016, 25, 095006.

15 L. N. Hao, Y. Chen and Y. S. Zhao, Mater. Res. Innovations, 2015, 19(suppl. 1), S1-477-S1-481.

16 K. Bian, H. Liu, G. Tai, K. Zhu and K. Xiong, J. Phys. Chem. C, 2016, 120, 12377-12384.

17 Inamuddin, A. Khan, M. Luqman and A. Dutta, Sens. Actuators, A, 2014, 216, 295-300. 
18 K. Tien Nguyen, S. Young Ko, J.-O. Park and S. Park, J. Mech. Robot., 2016, 8, 041006.

19 Y. Tang, Z. Xue, X. Zhou, X. Xie and C.-Y. Tang, Sens. Actuators, B, 2014, 202, 1164-1174.

20 S. Kim, S. Hong, Y.-Y. Choi, H. Song and K. No, Electrochim. Acta, 2013, 108, 547-553.

21 K. Surana, P. K. Singh, B. Bhattacharya, C. S. Verma and R. M. Mehra, Ceram. Int., 2015, 41, 5093-5099.

22 Q. He, L. Song, M. Yu and Z. Dai, Smart Mater. Struct., 2015, 24, 075001.

23 Y. Wang, H. Chen, Y. Wang, Z. Zhu and D. Li, Electrochim. Acta, 2014, 129, 450-458.

24 V. Palmre, S. J. Kim, D. Pugal and K. Kim, Int. J. Smart Nano Mater., 2014, 5, 99-113.

25 A. Aabloo, V. De Luca, G. Di Pasquale, S. Graziani, C. Gugliuzzo, U. Johanson, C. Marino, A. Pollicino and R. Puglisi, Sens. Actuators, A, 2016, 249, 32-44.

26 H.-Y. Jung and J. W. Kim, Int. J. Hydrogen Energy, 2012, 37, 12580-12585.

27 H.-Y. Jung, K.-Y. Cho, Y. M. Lee, J.-K. Park, J.-H. Choi and Y.-E. Sung, J. Power Sources, 2007, 163, 952-956.
28 S. H. de Almeida and Y. Kawano, J. Therm. Anal. Calorim., 1999, 58, 569-577.

29 S. J. Lee, M. J. Han, S. J. Kim, J. Y. Jho, H. Y. Lee and Y. H. Kim, Smart Mater. Struct., 2006, 15, 1217-1224.

30 G.-C. Cha, J.-S. Song, S.-M. Lee and M.-S. Mun, Polymer, 2006, 30, 471-477.

31 S.-G. Lee, H.-C. Park, S. D. Pandita and Y. Yoo, Int. J. Control Autom. Syst., 2006, 4, 748-755.

32 L. Vegard, Z. Phys., 1921, 5, 17-26.

33 M. R. VanLandingham, J. S. Villarrubia, W. F. Guthrie and G. F. Meyers, Macromol. Symp., 2001, 167, 15-44.

34 Q. S. Li, G. Y. H. Lee, C. N. Ong and C. T. Lim, Biochem. Biophys. Res. Commun., 2008, 374, 609-613.

35 Y.-R. Chang, V. K. Raghunathan, S. P. Garland, J. T. Morgan, P. Russell and C. J. Murphy, J. Mech. Behav. Biomed. Mater., 2014, 37, 209-218.

36 A. Fabre, S. Salameh, L. C. Ciacchi, M. T. Kreutzer and J. R. van Ommen, J. Nanopart. Res., 2016, 18, 200.

37 H. Kim, S. Lee, S. Kim, C. Oh, J. Ryu, J. Kim, E. Park, S. Hong and K. No, J. Mater. Sci., 2016, 52, 2400-2412.

38 X. Cheng, C. Peng, M. You, L. Liu, Y. Zhang and Q. Fan, Electrochim. Acta, 2006, 51, 4620-4625. 